\title{
W-type suture in Anderson-Hynes laparoscopic pyeloplasty: a novel approach to an old technique
}

\author{
Dawid Janczak ${ }^{1}$, Urszula Szydełko², Wojciech Apoznański ${ }^{3}$, Wojciech Panek ${ }^{1}$, Tomasz Szydełko \\ ${ }^{1}$ Department of Urology, $4^{\text {th }}$ Military Hospital, Wroclaw, Poland \\ ${ }^{2}$ Students Scientific Circle, Department of ENT, Head and Neck Surgery, Wroclaw Medical University, Wroclaw, Poland \\ ${ }^{3}$ Department of Pediatric Surgery and Urology, Wroclaw Medical University, Wroclaw, Poland
}

Videosurgery Miniinv 2018; 13 (4): 512-517

DOI: https://doi.org/10.5114/wiitm.2018.76087

\begin{abstract}
Introduction: Anderson-Hynes (A-H) dismembered pyeloplasty has remained nearly unchanged since its introduction in 1949. The authors present a modification of the uretero-pelvic anastomosis as described by Anderson and Hynes. The new approach, called the W-type suture, is thought to be more watertight and associated with fewer complications than the original.

Aim: To assess the effectiveness of the modification of Anderson-Hynes dismembered pyeloplasty named the W-type suture.

Material and methods: The research is a retrospective study of 99 patients who underwent laparoscopic $A-H$ pyeloplasty. In 49 patients unmodified laparoscopic $A-H$ pyeloplasty was carried out. Fifty patients underwent laparoscopic $A-H$ pyeloplasty with the anastomosis made using the aforementioned $W$-type suture. To assess the effectiveness of the W-type suture, we compared urine leakage duration - a direct indicator of anastomosis tightness - and frequency of postoperative complications.

Results: The mean duration of urinary leakage was $3 \pm 1.16$ days for the $W$-type suture anastomosis group and 3.57 \pm 1.14 days for the unmodified $\mathrm{A}-\mathrm{H}$ pyeloplasty group $(p<0.05)$. The frequency of post-operative complications did not statistically significantly differ between the groups except for fever (18.4\% vs. 4\%; $p<0.05$ ).

Conclusions: The analysis of the collected data showed that the W-type suture might be a promising alternative to a traditional approach of performing the ureteropelvic anastomosis. Further research should be done to minimize biases present in this study, which might have influenced our results.
\end{abstract}

Key words: pyeloplasty, anastomosis, Anderson-Hynes, dismembered.

\section{Introduction}

Since its introduction in 1949, the dismembered pyeloplasty technique invented by Anderson and Hynes $(\mathrm{A}-\mathrm{H})$ has been considered the gold standard in the treatment of ureteropelvic junction obstruction (UPJO) [1]. For most of the $20^{\text {th }}$ century it was performed as an open operation, but since the end of the last century, A-H pyeloplasty has been used as a minimally invasive procedure - the first laparoscopic pyeloplasty was done in 1993 by Schuessler et al., as well as by Kavoussi and Peters, and the first robotic approach was performed in 2002 by Gettman et al. [2-4]. Since then, minimally invasive pyeloplasty has become a well-studied, commonly used technique among urologists, with a success rate of over $90 \%[5,6]$.

Despite the rapid technological development in medicine and introduction of minimally invasive

\section{Address for correspondence}

Dawid Janczak MD, Department of Urology, $4^{\text {th }}$ Military Hospital, 5 Weigla St, 50-981 Wroclaw, Poland, phone: +48669989983 ,

e-mail: janczak.dawid@wp.pl 
techniques, the technique of performing the anastomosis during $\mathrm{A}-\mathrm{H}$ pyeloplasty has remained almost unchanged since 1949.

The authors present a modification to the old method, which they call the W-type suture. To assess the effectiveness of the W-type suture, we compared urine leakage duration - a direct indicator of anastomosis tightness - and frequency of complications between patients who underwent classic and modified A-H laparoscopic pyeloplasty.

\section{Aim}

To assess the effectiveness of the modification of Anderson-Hynes dismembered pyeloplasty named the W-type suture.

\section{Material and methods}

The data analyzed in the study are a part of a larger database of laparoscopic pyeloplasties collected by one of the authors between 2002 and 2015 (T.Sz.). The authors included in the study 49 patients who underwent an $\mathrm{A}-\mathrm{H}$ pyeloplasty with the unmodified suturing technique for the ureteropelvic anastomosis (between 2002 and 2007) - group 1, and 50 patients who underwent laparoscopic A-H pyeloplasty with the anastomosis made with the W-type technique (between 2011 and 2015) - group 2. Patients with no clear data regarding the suture technique used for the anastomosis and cases in which other modifications of anastomosis were used were excluded from this study.

All patients had undergone preoperative examinations such as ultrasonography (US), diuretic renography (DR), excretory intravenous urography (IVU) or computed tomography (CT) to confirm the diagnosis of UPJO. A four-grade scale was used to estimate the degree of hydronephrosis on the grounds of renal US or urography: grades 1 (mild) and 2 (moderate) were combined and analyzed as small - S; grades 3 (severe) and 4 (extreme) were combined and analyzed as large - L [7-9].

Pyeloplasty was carried out if the minimal split renal function on DR was above $15 \%$. It was as sumed that in nonobstructed pelvicaliceal systems the half time to tracer clearance (T1/2) was shorter than $12 \mathrm{~min}$. In the obstructed systems it was longer than $20 \mathrm{~min}$. The values between 12 and $20 \mathrm{~min}$ were equivocal.
Pain in all patients was evaluated on the basis of a visual analog pain scale (VAS). Patients with negative urine culture were administered only perioperative antibiotic treatment. Patients with positive urine culture were operated on while being treated with full-length targeted antibiotic therapy.

Postoperative complications were subdivided according to the Clavien-Dindo classification of surgical complications. The tightness of the anastomosis was evaluated in terms of the duration of drainage: the shorter the drainage time, the tighter the anastomosis. A drain was removed when the drainage was $<50 \mathrm{ml} /$ day.

The follow-up system was described in detail previously [9]. Briefly, the stent was removed 4 weeks after the surgery. Diuretic renography was performed 3, 13 and 25 months after the procedure. Ultrasonography and the assessment of symptoms were carried out every 3 months for the first 2 years after the surgery and then once a year. The mean follow-up was $73.3 \pm 16.6$ months in group 1 and 30.6 \pm 13.4 months in group 2 . The data regarding the duration of long-term follow-up of 1 patient from group 1 were missing.

A good final outcome was defined as symptom relief, significant reduction of hydronephrosis on US as well as no obstruction and improved or stable renal function on DR.

\section{W-type suture technique}

A detailed account of the operative technique was published previously [9].

Pyeloplasty was performed with the excision of the stenotic segment and, in patients with grade 3-4 hydronephrosis, with a reduction of the renal pelvis. The ureter was then spatulated, and the ureteropelvic reanastomosis was performed.

In group 1 the anastomoses were completed with interrupted sutures over a "double J" stent, using a freehand suturing technique. The preferred suturing material was 4-0 Vicryl (Ethicon, Johnson \& Johnson Intl, Belgium) with a curved needle. The apex of the spatulated part of the ureter was brought to the most dependent, inferior part of the renal pelvis with the first suture. The anastomosis was then performed with the interrupted sutures unless the spatulated part of the ureter was approximated to the inferior part of the renal pelvis. Then the cephalad aspect of the renal pelvis was closed 
with a running suture and the most inferior part of the pelvis was joined to the upper part of the ureter.

In an anastomosis performed in such a way a leakage between the inferior part of the pelvis and upper part of the ureter may occur.

In order to perform a more watertight anastomosis we modified our technique. All patients in group 2 were operated on with the modification. In all cases 4-0 monofilament Monocryl suture (Ethicon, Johnson \& Johnson Intl, Belgium) with a curved needle was used. The first running suture was placed from the apex of the spatulated ureter to the upper part of the dorsal border of the ureter through the ureteral and renal pelvic walls. The ventral border of the ureter was brought to the dependent part of the renal pelvis with the second running suture. With the same suture the upper, free part of the ureter was then approximated to the renal pelvis, going through the ureter from the outside to the inside, then through to the ureter from the inside to the outside, again through the ureter from the outside to the inside and through the renal pelvis wall from the inside to the outside, forming a W-shape. The remaining part of the renal pelvis was then closed in a running manner using the same suture (Figure 1).

\section{Statistical analysis}

The Fisher exact test was used to test categorical variables for statistical significance. The Shapiro-Wilk test was used to confirm whether numerical variables fit a normal distribution. If the data fit a normal distribution, an independent samples $t$ test was used to compare the numerical variables. If the data did not fit a normal distribution, the Mann-Whitney $U$-test was used to compare numerical variables. A $p$-value of less than 0.05 was considered statistically significant. The analysis was performed using Dell Statistica ver. 13.1. The data are presented as a frequency and percentage for the categorical variables or as a mean and standard deviation for the continuous variables.

\section{Results}

The two compared groups were similar regarding age, sex, UPJO side, presence of urolithiasis in affected kidney and occurrence of crossing vessels. Patients in the W-type suture group had had more procedures (i.e., percutaneous nephrostomy, insertion of DJ stent, ureterorenoscopy, endopyelotomy or other) performed on the kidney with UPJO prior to pyeloplasty (12 vs. 4 ) (24\% vs. $8.2 \%$ ) compared to the unmodified $\mathrm{A}-\mathrm{H}$ group, but the difference was not statistically significant $(p=0.054)$. A higher degree of hydronephrosis was less frequent in the unmodified A-H group (63.3\% vs. $84 \%$ ) and the difference was statistically significant $(p<0.05)$. The mean operative time was $217.73 \pm 50.9$ min for the classic $\mathrm{A}-\mathrm{H}$ group and $163.2 \pm 42.3 \mathrm{~min}$ for the $W$-type suture group $(p<0.05)$. The patients' data are presented in Table I.

The mean duration of urinary leakage was $3 \pm 1.16$ days for the W-type suture anastomosis group and $3.57 \pm 1.14$ days for the unmodified $\mathrm{A}-\mathrm{H}$ pyeloplasty group $(p<0.05)$. The frequency of post-operative complications did not statistically significantly differ between the groups except for fever (Clavien-Dindo - 1) $(18.4 \%$ vs. $4 \%$; $p<0.05)$. The final outcome was good in $96 \%$ in group 1 and $98 \%$ in group 2 . One patient's data regarding the outcome of the unmodified $\mathrm{A}-\mathrm{H}$ group were missing. No major or clinically significant complications occurred in either group (Table II).

\section{Discussion}

Since its introduction in 1949, Anderson-Hynes dismembered pyeloplasty has been the gold stan-
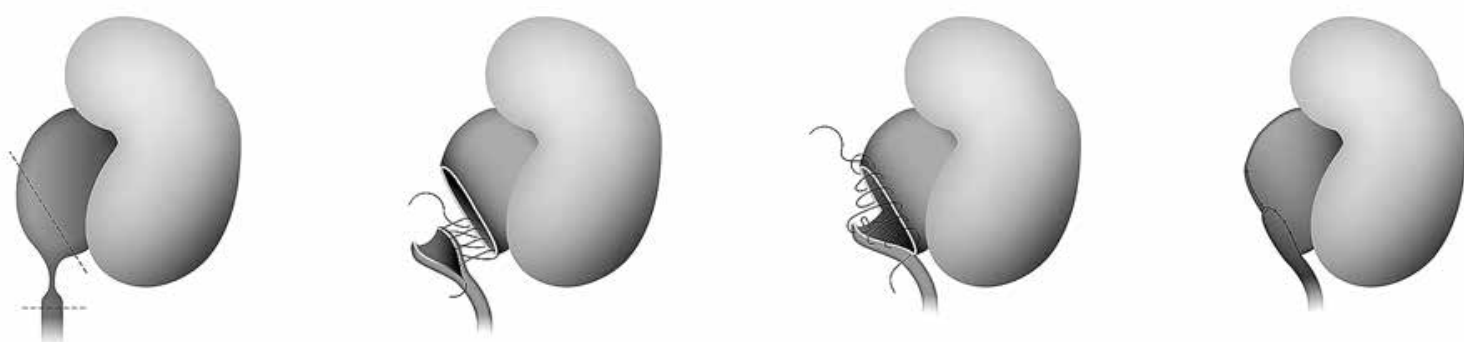

Figure 1. W-type suture technique 
Table I. Group characteristics

\begin{tabular}{|c|c|c|c|}
\hline Parameter & $\begin{array}{c}\text { Group } 1- \\
\text { A-H } \\
(n=49)\end{array}$ & $\begin{array}{c}\text { Group } 2- \\
\text { A-H-W } \\
(n=50)\end{array}$ & $P$-value \\
\hline Age & $31.8 \pm 12.2$ & $35.1 \pm 12.7$ & NS \\
\hline \multicolumn{4}{|l|}{ Sex: } \\
\hline Female & $25(51 \%)$ & $33(66 \%)$ & \multirow[t]{2}{*}{ NS } \\
\hline Male & $24(49 \%)$ & $17(34 \%)$ & \\
\hline \multicolumn{4}{|c|}{ Affected kidney treatment prior to pyeloplasty: } \\
\hline PCN & $3(6.1 \%)$ & $5(10 \%)$ & NS \\
\hline DJ & $1(2 \%)$ & $5(10 \%)$ & NS \\
\hline URS & 0 & $1(2 \%)$ & NS \\
\hline Endopyelotomy & 0 & $1(2 \%)$ & NS \\
\hline Total & $4(8.2 \%)$ & $12(24 \%)$ & NS \\
\hline $\begin{array}{l}\text { Urolithiasis in } \\
\text { affected kidney }\end{array}$ & $7(14.3 \%)$ & $4(8 \%)$ & NS \\
\hline \multicolumn{4}{|c|}{ Pelvis size (hydronephrosis): } \\
\hline $\mathrm{S}$ & $18(36.7 \%)$ & $2(4 \%)$ & \multirow[t]{3}{*}{$<0.05$} \\
\hline L & $31(63.3 \%)$ & $42(84 \%)$ & \\
\hline No data & 0 & $6(12 \%)$ & \\
\hline \multicolumn{4}{|l|}{ Side: } \\
\hline $\mathrm{R}$ & 21 (42.9\%) & $29(58 \%)$ & \multirow[t]{2}{*}{ NS } \\
\hline $\mathrm{L}$ & $28(57.1 \%)$ & $21(42 \%)$ & \\
\hline Operative time [min] & $\begin{array}{l}217.73 \\
\pm 50.9\end{array}$ & $\begin{array}{l}163.2 \\
\pm 42.3\end{array}$ & $<0.05$ \\
\hline \multicolumn{4}{|l|}{ Crossing vessels: } \\
\hline Yes & $23(46.9 \%)$ & $18(36 \%)$ & \multirow[t]{2}{*}{ NS } \\
\hline No & $26(53.1 \%)$ & $32(64 \%)$ & \\
\hline
\end{tabular}

A-H - unmodified A-H pyeloplasty group, A-H-W - W-type suture anastomosis group, $P C N$ - percutaneous nephrostomy, DJ - double J stent inserted, URS - ureterorenoscopy, $S$ - small, L - large, NS - p-value not significant.

dard of UPJO treatment. Initially performed as an open procedure, nowadays after the widespread adoption of minimally invasive techniques, it has become a method of choice for the laparoscopic approach as well. It has been proven that laparoscopic or robotic pyeloplasty is associated with a high success rate, better cosmetic results, reduced hospital stay and similar costs compared to an open operation [10].

A-H pyeloplasty, despite having been used for over 60 years, has not dated, and no other technique or modification has been commonly used. In 2015 Adam and Smith found that in the majority of publications $(10 / 17)$ on $\mathrm{A}-\mathrm{H}$ pyeloplasty the surgeons had
Table II. Outcomes

\begin{tabular}{|c|c|c|c|}
\hline Outcomes & $\begin{array}{c}\text { Group } 1- \\
\text { A-H } \\
(n=49)\end{array}$ & $\begin{array}{c}\text { Group } 2- \\
\text { A-H-W } \\
(n=50)\end{array}$ & $P$-value \\
\hline \multicolumn{4}{|c|}{ Drain removal [days]: } \\
\hline 1 & 1 & 0 & \multirow[t]{8}{*}{$<0.05$} \\
\hline 2 & 3 & 24 & \\
\hline 3 & 24 & 10 & \\
\hline 4 & 14 & 9 & \\
\hline 5 & 4 & 6 & \\
\hline 6 & 1 & 1 & \\
\hline 7 & 2 & 0 & \\
\hline Mean & $3.57 \pm 1.14$ & $3 \pm 1.16$ & \\
\hline \multicolumn{4}{|c|}{ Post-op. complications: } \\
\hline DJ obstruction & $2(4.1 \%)$ & $4(8 \%)$ & NS \\
\hline Fever & 9 (18.4\%) & $2(4 \%)$ & $<0.05$ \\
\hline PCN post-op. & $4(8.2 \%)$ & $2(4 \%)$ & NS \\
\hline Other & $5(10.2 \%)$ & $4(8 \%)$ & NS \\
\hline Total & $20(40.8 \%)$ & $12(24 \%)$ & NS \\
\hline \multicolumn{4}{|l|}{ Clavien-Dindo: } \\
\hline 1 & 12 & 4 & $<0.05$ \\
\hline 2 & 1 & 1 & \multirow[t]{3}{*}{ NS } \\
\hline 3 & 7 & 7 & \\
\hline 4 & 0 & 0 & \\
\hline \multicolumn{4}{|l|}{ Outcome: } \\
\hline Good & 47 (96\%) & 49 (98\%) & \multirow[t]{3}{*}{ NS } \\
\hline Bad & $1(2 \%)$ & $1(2 \%)$ & \\
\hline No data & $1(2 \%)$ & 0 & \\
\hline
\end{tabular}

A-H - unmodified $\mathrm{A}-\mathrm{H}$ pyeloplasty group, $\mathrm{A}-\mathrm{H}-\mathrm{W}-\mathrm{W}$-type suture anastomosis group, DJ - double J stent, PCN post-op. - percutaneous nephrostomy performed postoperatively, NS - not significant $p$-value.

in fact used their own modification of the A-H procedure, even though the technique was described as unmodified. For that reason, in order to make any comparison of the results possible and reliable, Adam and Smith proposed a classification for dismembered pyeloplasty based on the types of variations of a pelvic incision [11]. According to this classification, both groups compared in our study had their renal pelvis incised not with the original L-shaped cut, as described by Anderson and Hynes, but with an oblique renal pelvis cut.

Minimally invasive $\mathrm{A}-\mathrm{H}$ pyeloplasty is a technically challenging procedure, which requires advanced surgical skills and has a steep learning curve. The 
most demanding part is performing the anastomosis. Qu et al. presented their modification of dismembered pyeloplasty that is supposed to decrease the difficulty of surgery while maintaining $97.8 \%$ of the success rate [12]. The uretero-pelvic anastomosis was made between the posterior part of the spatulated ureter and the anterior surface of the renal pelvis. Their approach is described as a method facilitating suturing, but Qu et al. did not compare the outcomes of the modified approach to the results of standard Anderson-Hynes pyeloplasty achieved by the same surgeons.

The authors of this article have not found any English publication on modifications of laparoscopic, adult $\mathrm{A}-\mathrm{H}$ pyeloplasty comparing the results of the modified method with the original one. A search was performed on the PubMed database using the term "Anderson-Hynes pyeloplasty".

The analysis of our data has shown that our modification of the suturing technique proved to be statistically significantly superior to the unmodified technique in terms of urine leakage duration. The drain was removed within 2 days postoperatively in $48 \%$ ( 0 on day 1, 24 on day 2, out of 50 patients) of patients in the study group. The use of the W-type suture may potentially reduce the overall therapy cost by shortening the hospital stay, thereby increasing patients' satisfaction while maintaining a very good treatment outcome.

The analysis also revealed a difference between groups in frequency of fever occurrence. Despite it being statistically significant, the authors believe that fever is multifactorially conditioned and the modification of the surgical technique may not necessarily be associated with fever occurrence. On the contrary, it seems to us that the earlier removal of the drainage, which is a potential source of infection, might have had an impact on lower fever occurrence in the W-type suture group.

The major limitations of our study are the length of the data collection period and retrospective nature of the analysis. All procedures were performed by experienced surgeons, but certainly the skill of the surgeon who conducted all operations using a modified suturing technique (T.Sz.), was gradually increasing between 2007 and 2011 as he was, throughout that period, a surgically active urologist. Even though patients received the same pre- and post-operative care, as the procedures remained unchanged, an overall constant improvement in medi- cal care (better equipment, implementation of new ISO standards) may have influenced the results. Also another factor which might have had an impact on the outcomes, especially on the operating time and tightness of the anastomosis, is the use of interrupted sutures in group 1 and a continuous suture in group 2. Despite a significant difference in the length of the follow-up periods between the groups, we do not consider it a major limitation as the study does not focus on long-term outcomes, but on the watertightness of the anastomosis.

\section{Conclusions}

Our study has shown that the W-type suture might be a promising alternative to a traditional approach of performing a uretero-pelvic anastomosis during $\mathrm{A}-\mathrm{H}$ pyeloplasty. It may reduce the duration of post-operative abdominal drainage, which is associated with a shorter hospital stay and consequent lower costs of the treatment. Further research should be done to minimize the limitations of our analysis.

\section{Conflict of interest}

The authors declare no conflict of interest.

\section{References}

1. Anderson JC, Hynes W. Retrocaval ureter: a case diagnosed pre-operatively and treated successfully by a plastic operation. Br J Urol 1949; 21: 209-14.

2. Schuessler WW, Grune MT, Tecuanhuey LV, Preminger GM. Laparoscopic dismembered pyeloplasty. J Urol 1993; 150: 1795-9.

3. Gettman MT, Neururer R, Bartsch G, Peschel R. Anderson-Hynes dismembered pyeloplasty performed using the da Vinci robotic system. Urology 2002; 60: 509-13.

4. Kavoussi LR, Peters CA. Laparoscopic pyeloplasty. J Urol 1993; 150: 1891-4.

5. Autorino R, Eden C, El-Ghoneimi A, et al. Robot-assisted and laparoscopic repair of ureteropelvic junction obstruction: a systematic review and meta-analysis. Eur Urol 2014; 65: 430-52.

6. Strother MC, Mucksavage P. Minimally invasive techniques for the management of adult UPJ obstruction. Curr Urol Rep 2016; 17: 39.

7. Talner L. Urinary obstruction. In: Clinical Urography: An Atlas and Textbook of Urological Imaging. Vol. 2. W.B. Saunders 1990; 1535-628.

8. Fernbach SK, Maizels M, Conway JJ. Ultrasound grading of hydronephrosis: introduction to the system used by the society for fetal urology. Pediatr Radiol 1993; 23: 478-80.

9. Szydełko T, Kasprzak J, Apoznański W, et al. Comparison of dismembered and nondismembered $\mathrm{Y}$-V laparoscopic pyeloplasty 
in patients with primary hydronephrosis. J Laparoendosc Adv Surg Tech A 2010; 20: 7-12.

10. Jacobs BL, Seelam R, Lai JC, et al. Cost analysis of treatments for ureteropelvic junction obstruction. J Endourol 2017; 31: 204-9.

11. Adam A, Smith GHH. Anderson-Hynes pyeloplasty: are we all really on the same page? ANZ I Surg 2016; 86: 143-7.

12. Ou ZY, Chen JB, Chen Z, et al. Retroperitoneoscopic dismembered pyeloplasty for ureteropelvic junction obstruction: modification of the procedure and our experience. Urol I 2014; 11 1763-767.

Received: 22.03.2018, accepted: 12.04.2018. 